\title{
Probabilistic Load Flow Considering Wind Generation Uncertainty
}

\author{
Morteza Aien \\ Electrical Engineering Dpt \\ Sharif University of Technology \\ Tehran, Iran \\ morteza_aien@yahoo.com
}

\author{
Reza Ramezani \\ Electrical Engineering Dpt \\ Sharif University of Technology \\ Tehran, Iran \\ rezaramezani65@gmail.com
}

\author{
S. Mohsen Ghavami \\ Shahid Bahonar Copper Industries \\ (SBCI) \\ Kerman, Iran \\ mohsen.ghavami270@gmail.com
}

\begin{abstract}
Renewable energy sources, such as wind, solar and hydro, are increasingly incorporated into power grids, as a direct consequence of energy and environmental issues. These types of energies are variable and intermittent by nature and their exploitation introduces uncertainties into the power grid. Therefore, probabilistic analysis of the system performance is of significant interest. This paper describes a new approach to Probabilistic Load Flow (PLF) by modifying the Two Point Estimation Method (2PEM) to cover some drawbacks of other currently used methods. The proposed method is examined using two case studies, the IEEE 9-bus and the IEEE 57-bus test systems. In order to justify the effectiveness of the method, numerical comparison with Monte Carlo Simulation (MCS) method is presented. Simulation results indicate that the proposed method significantly reduces the computational burden while maintaining a high level of accuracy. Moreover, that the unsymmetrical 2PEM has a higher level of accuracy than the symmetrical 2PEM with equal computing burden, when the Probability Density Function (PDF) of uncertain variables is asymmetric.
\end{abstract}

Keywords- probabilistic load flow; two point estimation method; uncertainty; wind turbine generator (WTG).

\section{NOMENCLATURE}

The notation used throughout the paper is stated below for quick reference.

\footnotetext{
c The weibull scale factor $[\mathrm{m} / \mathrm{s}]$.

$h \quad$ The nonlinear function that relates $Y$ to $X$, here, the LF problem.

$k \quad$ The weibull shape factor.

$M_{3}^{\prime}\left(X_{k}\right)$ 3th moment of the $x_{k}$ PDF..

$n \quad$ The number of uncertain variables.

$p_{k, 1} \quad$ The weight associated to $\varepsilon_{k, 1}$.

$p_{k, 2} \quad$ The weight associated to $\varepsilon_{k, 2}$.

$P \quad$ The wind turbine generator (WTG) output power [ $M W$ ].

$P_{D} \quad$ The demand active power [ $p u$ ].

$P_{D G} \quad$ The distributed active power generation [ $p u$ ].
}

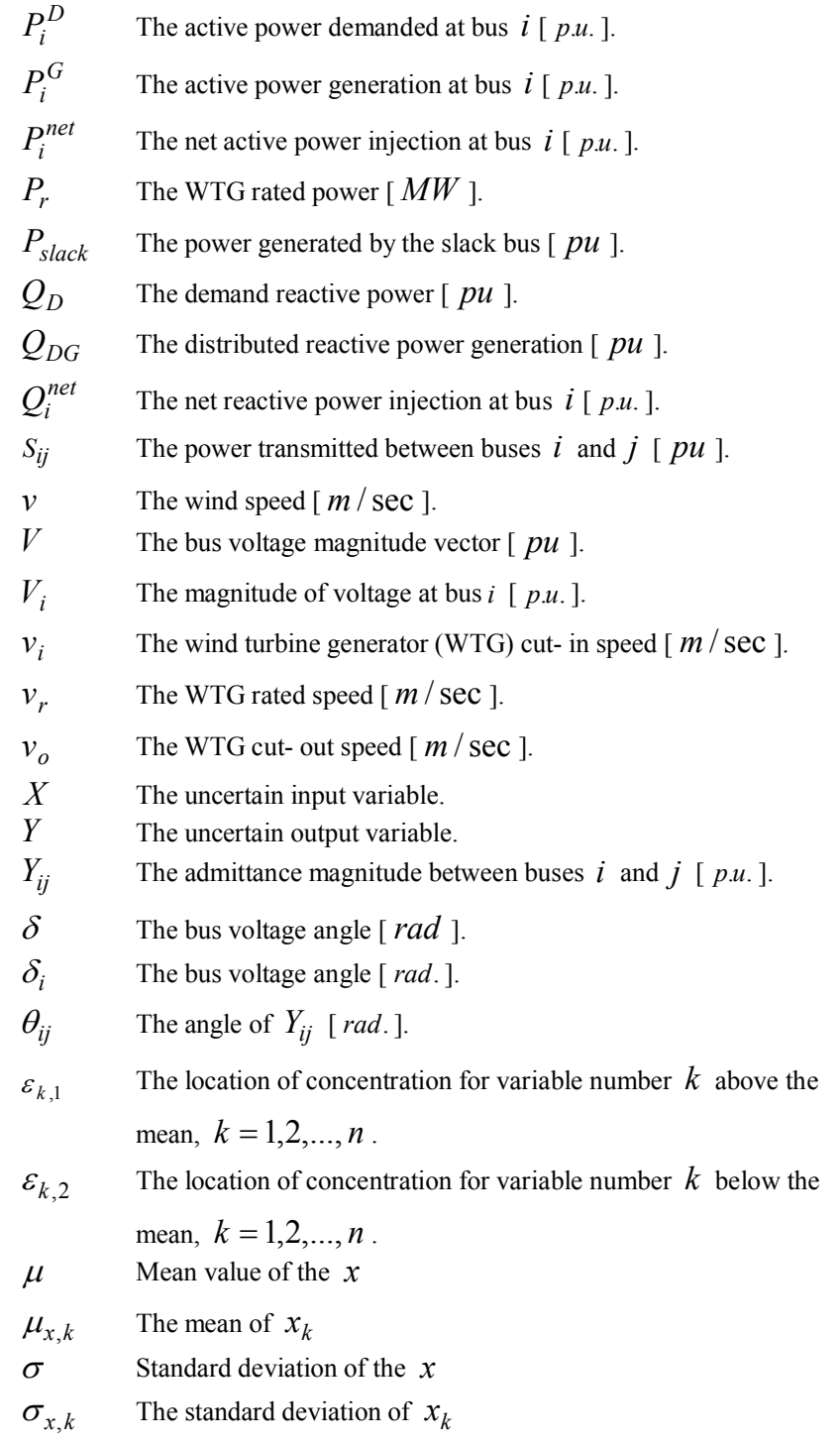




\section{INTRODUCTION}

\section{A. Motivation}

Computation of power flow in the electric power system is one of the major requirements that power system designers face. Deterministic load flow requires specific values for loads, unit's generation and network conditions. As the power industry is being restructured and new technologies develop more and more uncertainties are introduced. These uncertainties mainly stem from bus loads and changes in the network's configuration and power supplies. Recently, attention is particularly focused in the increasing penetration of renewable energy sources in power systems. The introduced uncertainties inevitably result to errors in the deterministic Load Flow (LF) solutions. In order to cope with the increased uncertainties imposed to power systems, probabilistic tools are employed to predict the state of the system at any time instant. In system planning, it is desired to assess bus voltages and line flows for a range of load and generation conditions. Executing the LF for every possible or probable combination of bus loads and generating unit's conditions is completely impractical because of the extremely large computational burden required. Performing PLF study gives system planning engineers a better feel of system conditions and can provide increased assurance regarding investment planning [1].

\section{B. Literature Review and Paper Contribution}

Several methods of analysis for engineering systems under uncertainty have been employed. To account for power system uncertainties, probabilistic techniques have been used since the early seventies [1], when the uncertainty in system demand was first considered. The Monte Carlo Simulation (MCS) technique has been employed to solve the PLF problem by repeated simulations and provided considerably accurate results with the drawback of very large computation time [2]. An approach based on the application of the Combined Cumulant and GramCharlier expansion theory in the PLF problem has been followed in $[3,4]$. The method has also been used in AC-PLF to compute the load flow of power system containing wind farms [5] and to obtain the PDF of transmission line flows [3]. Fast Fourier Transforms (FFT) and a discrete frequency domain convolution technique have been applied to reduce the computation time in [6]. A fuzzy concept to consider the wind generation uncertainties in the PLF problem has been employed in [7]. A two point estimation method (2PEM) has been applied to cope with uncertainties in engineering systems in $[8,9]$ and a point estimation method (PEM) has been used for PLF evaluation, taking into account the uncertainties of bus injections and line parameters in [10].

In this paper, a new method for PLF study is proposed, by adapting the 2 PEM to the PLF problem, taking into account the uncertainties of load, wind power generation and a simple branch outage. Different scenarios are defined and the obtained results are discussed for each case study. In order to justify the effectiveness of the method, the obtained results are compared with those of MCS in terms of accuracy and computational burden.

\section{Paper Organization}

Section II formulates the LF and the PLF problem. Section III introduces different PLF methods. Section IV describes the adaptation of the PLF methods to power systems. Section V reports results concerning two detailed case studies. Section VI presents a comparison of the results. Section VII closes the paper, providing concluding remarks. The Appendix clarifies technical details about the wind turbines used.

\section{LOAD FLOW (LF) PROBLEM}

LF computation is one of the most essential tasks in electric power systems. To handle this task successfully, the set of system state variables which contains the bus voltage magnitude and angles have to be known. Having this set of variables, every output variable can be calculated. The below section formulates the LF problem.

\section{A. Load Flow Formulation}

The LF problem is solved to obtain the power system state variables. The LF problem has been formulated in several technical references and will not be discussed at length here. However, it should be recalled that it can be formulated as follows:

$$
\begin{aligned}
P_{i}^{n e t} & =\sum_{G} P_{i}^{G}-\sum_{D} P_{i}^{D} \\
Q_{i}^{n e t} & =\sum_{G} Q_{i}^{G}-\sum_{D} Q_{i}^{D} \\
P_{i}^{n e t} & =V_{i} \sum_{j=1}^{N_{b}} Y_{i j} V_{j} \cos \left(\delta_{i}-\delta_{j}-\theta_{i j}\right) \\
Q_{i}^{\text {net }} & =V_{i} \sum_{j=1}^{N_{b}} Y_{i j} V_{j} \sin \left(\delta_{i}-\delta_{j}-\theta_{i j}\right)
\end{aligned}
$$

\section{B. PLF Formulation}

Power system has an uncertain nature. This causes power system analysis tools to be inaccurate when deterministic data are used, since the state of the system is changing instantaneously. This is why probabilistic data are used. The PLF problem is formulated as:

$$
Y=h(X)
$$

The input vector $X$ can be written as:

$$
X=\left[P_{D}, Q_{D}, P_{D G}, Q_{D G}, \ldots\right]
$$

And the output vector $Y$ can be written as:

$Y=\left[\delta, V, S_{i j}, P_{\text {slack }}, \ldots\right]$

\section{PLF METHODS}

Several methods for PLF study have been developed. Theses methods fall in two basic groups: simulation methods like MCS and analytical methods like 2PEM. 


\section{A. Monte Carlo Simulation Method}

MCS is a technique that involves using random numbers and probabilities to solve a probabilistic problem. It is a method for iteratively evaluating a deterministic model using sets of random numbers as inputs. This method is often used when the model is complex, nonlinear, or involves more than just a couple of uncertain parameters [11]. A simulation can typically involve over 10000 evaluations of the model, a task which in the past was only practical using super computers. Although the MCS method is able to provide accurate results, the computation is significantly time-consuming; therefore, it is not suitable for real time applications.

\section{B. Two Point Estimate Method}

In order to account for uncertainties in the PLF, a 2PEM [8], which is basically a variation of the original point estimate method (PEM) described in $[9,10]$, is used to decompose (5) into several sub-problems by taking only two deterministic values of each uncertain variable placed on both sides of the corresponding mean value. The deterministic LF is then ran twice for each uncertain variable, once for the value below the mean, and once for the value above the mean, with other variables kept at their means. These two points may be symmetric about the mean or not. Both possible point locations are investigated in this paper and simulation results are compared. In PLF studies it is desired to find the distribution function of output variables. Therefore, it is necessary to calculate the expected value and the standard deviation of the output variables using proper methods, such as 2PEM. The procedure of this method with symmetrical location of two sampling points is as follows [12]:

Step 1: determine the number of uncertain variables.

Step 2: set $E(Y)=0$ and $E\left(Y^{2}\right)=0$.

Step 3: set $k=1$.

Step 4: determine $\varepsilon_{k, 1}, \varepsilon_{k, 2}, p_{k, 1}$ and $p_{k, 2}$.

$\varepsilon_{k, 1}=\sqrt{n}$

$\varepsilon_{k, 2}=-\sqrt{n}$

$p_{k, 1}=p_{k, 2}=\frac{1}{n}$

Step 5: determine the two concentrations $\chi_{k, 1}$ and $\chi_{k, 2}$.

$$
\chi_{k, 1}=\mu_{x, k}+\varepsilon_{k, 1} \sigma_{x, k}
$$

$\chi_{k, 2}=\mu_{x, k}+\varepsilon_{k, 2} \sigma_{x, k}$

Step 6: run the deterministic LF for both concentrations $x_{k, i}$ using $X=\left[\mu_{x, 1}, \mu_{x, 2}, \ldots, \chi_{k, i}, \ldots, \mu_{x, n}\right], i=1,2$.

Step 7: calculate $E(Y)$ and $E\left(Y^{2}\right)$

$$
E(Y) \cong \sum_{k=1}^{n} \sum_{i=1}^{2}\left(P_{k, i} h\left(\left[\mu_{x, 1}, \mu_{x, 2}, \ldots, \chi_{k, i}, \ldots, \mu_{x, n}\right]\right)\right)
$$

$$
E\left(Y^{2}\right) \cong \sum_{k=1}^{n} \sum_{i=1}^{2}\left(P_{k, i} h\left(\left[\mu_{x, 1}, \mu_{x, 2}, \ldots, \chi_{k, i}, \ldots, \mu_{x, n}\right]\right)^{2}\right)
$$

Step 8: calculate the mean and the standard deviation of the output variable.

$$
\begin{aligned}
\mu_{Y} & =E(Y) \\
\sigma_{Y} & =\sqrt{E\left(Y^{2}\right)-E^{2}(Y)}
\end{aligned}
$$

It is obvious that the heart of the suggested method lies in how to produce appropriate samples having enough information about the PDF of input variables. In the case of unsymmetrical probability distributions for the input variables, the equations for the location of each point are [12]:

$$
\begin{array}{ll}
\varepsilon_{k, 1}=\frac{\lambda_{k, 3}}{2}+\sqrt{n+\left(\frac{\lambda_{k, 3}}{2}\right)^{2}}, & k=1,2, \ldots, n \\
\varepsilon_{k, 2}=\frac{\lambda_{k, 3}}{2}-\sqrt{n+\left(\frac{\lambda_{k, 3}}{2}\right)^{2}}, & k=1,2, \ldots, n \\
p_{k, 1}=-\frac{\varepsilon_{k, 2}}{n \varsigma_{k}} & \\
p_{k, 2}=\frac{\varepsilon_{k, 1}}{n \varsigma_{k}} & k=1,2, \ldots, n \\
\varsigma_{k}=2 \sqrt{n+\left(\lambda_{k, 3} / 2\right)^{2}}, & \\
\sum_{k=1}^{n}\left(p_{k, 1}+p_{k, 2}\right)=1 & \\
p_{k, 1}+p_{k, 2}=\frac{1}{n} & \\
\lambda_{k, 3}=\frac{M_{3}^{\prime}\left(x_{k}\right)}{\sigma_{x, k}^{3}} &
\end{array}
$$

It is notable that for symmetric probability distributions, in which skewness and as a result $\lambda_{k, 3}$ equals to zero, (17)-(20) convert to (8)-(10). Finally, the first three moments can then be approximated by:

$$
\begin{aligned}
& E(Y) \cong \sum_{k=1}^{n} \sum_{i=1}^{2}\left(P_{k, i} h\left(\left[\mu_{x, 1}, \mu_{x, 2}, \ldots, \chi_{k, i}, \ldots, \mu_{x, n}\right]\right)\right) \\
& E\left(Y^{2}\right) \cong \sum_{k=1}^{n} \sum_{i=1}^{2}\left(P_{k, i} h\left(\left[\mu_{x, 1}, \mu_{x, 2}, \ldots, \chi_{k, i}, \ldots, \mu_{x, n}\right]\right)^{2}\right) \\
& E\left(Y^{3}\right) \cong \sum_{k=1}^{n} \sum_{i=1}^{2}\left(P_{k, i} h\left(\left[\mu_{x, 1}, \mu_{x, 2}, \ldots, \chi_{k, i}, \ldots, \mu_{x, n}\right]\right)^{3}\right)
\end{aligned}
$$

\section{ADAPTATION OF THE PLF METHODS TO THE POWER SYSTEM}

For probabilistic modeling, the uncertain variables must be specified first. The next step is uncertainty modeling and the final step is evaluating the output variable statistics. The uncertain parameters related to the power system include generation, load, and network topology. This paper mostly focuses on the load, wind power generation and simple network configuration uncertainties. The load in each bus is modeled with a normal PDF with $\mu$ equal to the base load and $\sigma$ equal to $\pm 5 \%$ of the base load. The normal PDF can be stated as (28). 
$f(x)=\frac{1}{\sqrt{2 \pi} \sigma} e^{\left[-\frac{1}{2}\left(\frac{x-\mu}{\sigma}\right)^{2}\right]}$

The wind speed is modeled with weibull PDF given by:

$$
f(v)=\frac{k}{c}\left(\frac{v}{c}\right)^{k-1} e^{\left[-\left(\frac{v}{c}\right)^{k}\right]}
$$

It is assumed that in each region, the PDF of wind speed is known, therefore, the transformation of wind speed to wind turbine output power is given by:

$\begin{cases}P=0, & v \leq v_{i} \text { or } v>v_{o} \\ P=P_{r} \frac{v-v_{i}}{v_{r}-v_{i}}, & v_{i}<v<v_{o} \\ P=P_{r}, & v_{i}<v<v_{o}\end{cases}$

The system loads are modeled as PQ buses with specified real and reactive load demand and the wind farm output power is modeled as negative load. For simple modeling of the network topology, some transmission lines have been assumed to have a specific Forced Outage Rate (FOR). The overall procedure of PLF evaluation is as follows: in each iteration, the quantity of uncertain input parameters is obtained, the LF problem is executed and the output variables are stored. Finally, the statistics of the uncertain output variables are calculated using (13)-(16) or other appropriate equations such as (31)-(32) depending on the method used.

\section{CASE STUDIES}

To justify the effectiveness of the proposed method, two case studies are employed, a 9-bus, and a 57-bus standard system, and the obtained results are compared with those obtained by MCS. The proposed method was implemented on a Dell Inspiron 1420 system with a 2-GHz processor and 2-GB of RAM using MATLAB optimization toolbox.

\section{A. Case Study 1}

This case study is the IEEE 9-bus system with base power, base voltage, and total active and reactive demand equal to $100 \mathrm{MVA}, 345 \mathrm{kV}, 315 \mathrm{MW}$ and $115 \mathrm{MVAr}$, respectively. The single line diagram of this network is illustrated in Figure 1. In this case, bus 7 is assumed to have a wind farm whose detailed information is attached in the Appendix.

In this case, the point of interest is to investigate on the total active losses [MW], $P_{6 \rightarrow 7}$ (power from bus6 to bus 7 [MW]) and $P_{7 \rightarrow 8}$ (power from bus 7 to bus 8 [MW]). In the PLF study, it is desired to assess the contribution of each uncertainty on the output variables uncertainty. To do this, four scenarios are defined:

Scenario 1: the load is assumed fixed and active power generation of the wind farm is considered as an uncertain variable.
Scenario 2: In this scenario, besides the wind farm output power uncertainty, the load is also assumed as another uncertain variable.

Scenario 3: In addition to uncertainties of scenario 2, in the third scenario the lines between buses 6,7 and buses 7, 8 are assumed to have $\mathrm{FOR}=0.05$. It must be noted that these lines connect bus 7 , in which the wind farm is placed, to the other buses.

Scenario 4: In addition to the assumptions of the third scenario, the reactive power consumption of wind farm is also considered with a power factor equal to 0.85lag.

Table I outlines the summary of the assumptions for each scenario. It is noteworthy that there is a base scenario in which none of the uncertainties mentioned above exist. In Table I, Y and $\mathrm{N}$ denote Yes or No, respectively.

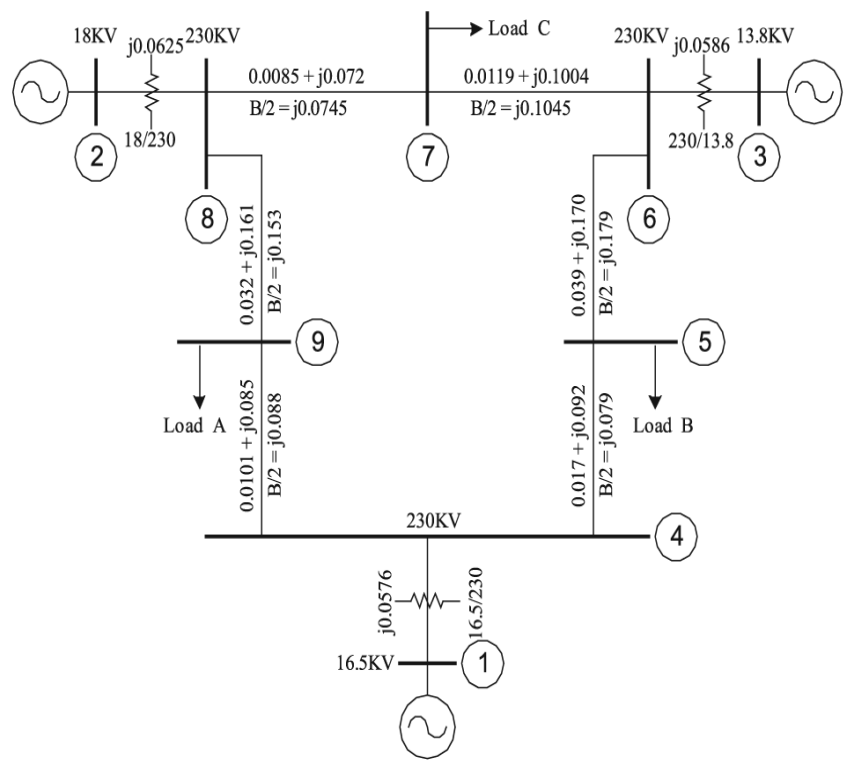

Fig. 1. Single line diagram of 9-bus test system.

TABLE I. SUMMARY OF DIFFERENT SCENARIOS

\begin{tabular}{|c|c|c|c|c|}
\hline Scenarios & $\begin{array}{c}\text { WIND GEN. } \\
\text { UNCERT. }\end{array}$ & $\begin{array}{c}\text { LOAD } \\
\text { UNCERT. }\end{array}$ & $\begin{array}{c}\text { Lines } \\
\text { FOR }\end{array}$ & $\begin{array}{c}\text { WIND FARM Q } \\
\text { CONSUM. }\end{array}$ \\
\hline Base & N & N & N & N \\
\hline No.1 & Y & N & N & N \\
\hline No.2 & Y & Y & N & N \\
\hline No.3 & Y & Y & Y & N \\
\hline No.4 & Y & Y & Y & Y \\
\hline
\end{tabular}

In the MSC method, the statistics of output variables can be obtained using (31) - (32).

$$
\begin{aligned}
\mu_{M C S} & =\frac{1}{N} \sum_{i=1}^{N} x_{i} \\
\sigma_{M C S} & =\sqrt{\frac{1}{N} \sum_{i=1}^{N}\left(x_{i}-\mu_{M C S}\right)^{2}}
\end{aligned}
$$


where $\mathrm{N}$ is the number of MCS samples, which in this study is set to 3000 samples and $\mathrm{x}$ is the output variable.

Table II shows the results for different scenarios in this case study. In Table II, the STD denotes the standard deviation of the output variable. The obtained results indicate that as the network uncertainty increases, the STD of output variables increases. The STD represents the degree of uncertainty for each variable. Increasing the input variables uncertainty increases the output variables uncertainty. STD is largest in the last scenario since this scenario has most uncertainty. The contents of Table II indicate that in this case, it is mostly the network configuration that affects the line flows, followed by the wind farm output power and load uncertainty.

Figures 2-6 show results using the theory of normal distribution function. The uncertainty can be better described by using the Cumulative Density Function (CDF) concept. Figures 5 and 6 portray the CDF of some output variables. As uncertainty increases, the slope of the CDF decreases as shown in Figure 5 and 6 . Figure 5 shows that the unsymmetrical 2PEM has a higher level of accuracy compared to the symmetrical 2PEM. It is also shown that in scenario 4, which has the most uncertainties, the CDF slope is smaller. The power transmitted from bus 6 to bus 7 in scenarios 3 and 4 may have reverse direction due to network configuration and wind generation uncertainties as shown in Figure 2. As shown in Figure 4, in case of total losses, the probabilities of being greater than $5 \mathrm{MW}$ in all scenarios are $0.7635,0.72,0.629$, and 0.633 , respectively. Table III compares the obtained results for scenario 2.

\section{B. Case Study 2}

This case is the IEEE 57-bus standard system with base MVA, base $\mathrm{kV}$, total active and reactive load equal to 100MVA, $345 \mathrm{kV}, 1250.8 \mathrm{MW}$ and 336.4MVAr, respectively. In this case, bus 8 is assumed to have a wind farm that its detailed information is attached in the Appendix. In this case study, scenario 4 which has the most uncertainty is evaluated by different methods and the obtained results are given in Table IV. Figure 7 shows the comparison of the CDF for total active losses obtained by different methods for this case study.

TABLE II. MEAN AND STD OF OUTPUT VARIABLES IN DIFFERENT SCENARIOS BY MCS - CASE1

\begin{tabular}{|c|c|c|c|c|c|}
\hline Scenarios & BASE & No.1 & No.2 & No.3 & No.4 \\
\hline Mean Active losses & 4.955 & 5.1097 & 5.1473 & 5.4388 & 5.5809 \\
\hline Std Active losses & 0 & 0.1523 & 0.2501 & 1.3251 & 1.7072 \\
\hline Mean $\mathrm{P}_{6-7}$ & 24.11 & 22.098 & 22.092 & 22.017 & 24.804 \\
\hline Std $\mathrm{P}_{6-7}$ & 0 & 1.906 & 3.0916 & 15.186 & 18.161 \\
\hline Mean $\mathrm{P}_{7-8}$ & -75.99 & -73.753 & -73.725 & -72.929 & -70.722 \\
\hline Std $\mathrm{P}_{7-8}$ & 0 & 2.123 & 3.4935 & 14.889 & 17.612 \\
\hline
\end{tabular}

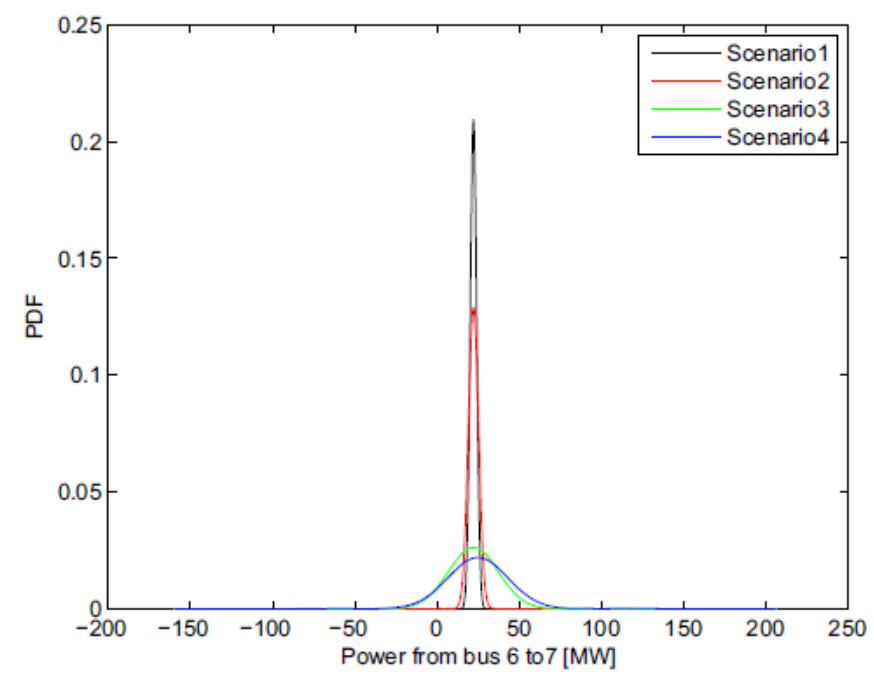

Fig. 2. The PDF of active power between buses 6 and 7- Case1.

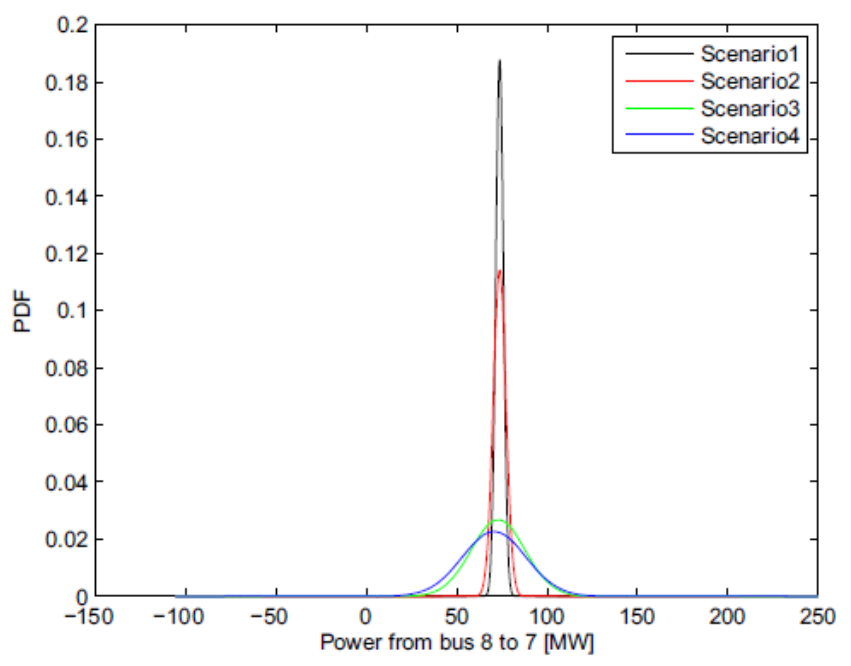

Fig. 3. The PDF of active power between buses 8 and 7- Case1.

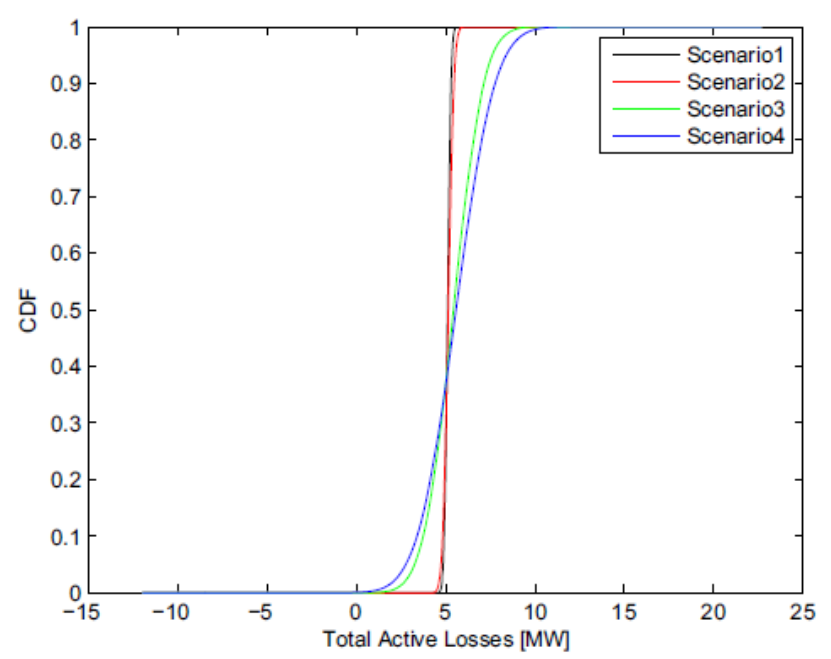

Fig. 4. The CDF of total active losses in different scenarios- Case1 


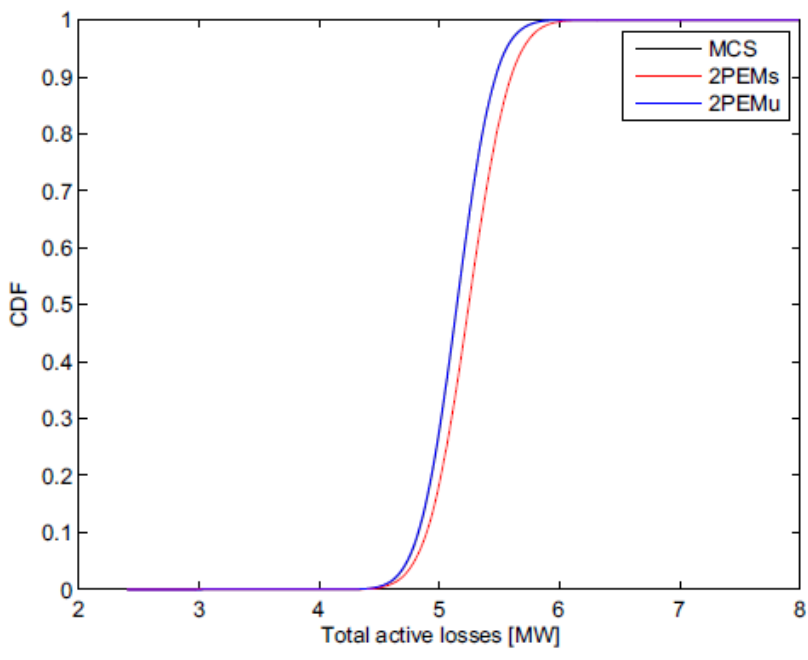

Fig. 5. The CDF of total active losses obtained by all methods- Case1.

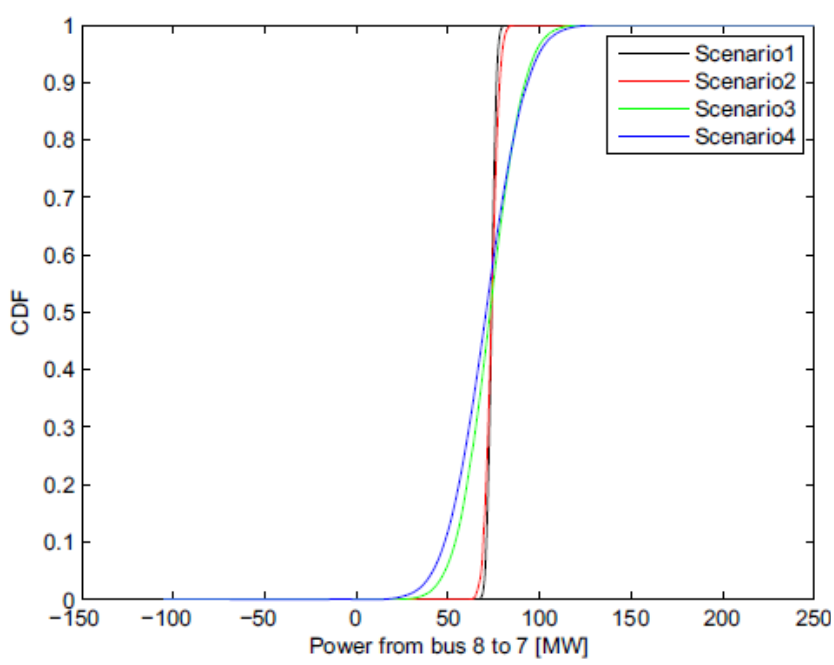

Fig. 6. CDF of active power between buses 8 and 7-Case1.

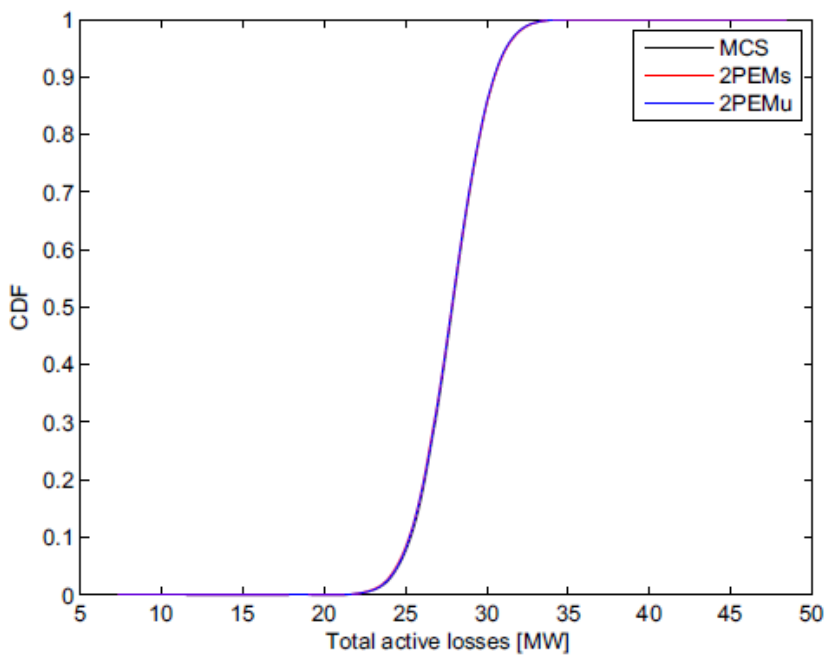

Fig. 7. The CDF of total active losses obtained by all methods-Case2.
TABLE III. COMPARISON OF THE RESULTS- CASE1

\begin{tabular}{|c|c|c|}
\hline Methods & MEAN OF THE LOSSES & STD OF THE LOSSES \\
\hline MCS & 5.1473 & 0.2501 \\
\hline 2 PEMs & 5.2452 & 0.2743 \\
\hline 2 PEMu & 5.1453 & 0.252 \\
\hline
\end{tabular}

TABLE IV. COMPARISON OF THE RESULTS- CASE2

\begin{tabular}{|c|c|c|}
\hline Methods & MEAN OF THE LOSSES & STD OF THE LOSSES \\
\hline MCS & 27.8841 & 2.01 \\
\hline $2 \mathrm{PEMs}$ & 27.83 & 2.052 \\
\hline $2 \mathrm{PEMu}$ & 27.8336 & 2.0244 \\
\hline
\end{tabular}

VI. COMPARISON OF THE RESULTS

The intention at this point is to interrogate the accuracy of the obtained results by $2 \mathrm{PEM}$; the mean and the standard deviation of results obtained by the 2PEM are compared with the corresponding values obtained by the MCS, which is considered accurate. The errors for the mean and standard deviation of output variable are respectively defined as:

$$
\begin{gathered}
\varepsilon_{\mu}=\left(\frac{\mu_{M C S}-\mu_{2 P E M}}{\mu_{M C S}}\right) \times 100 \\
\varepsilon_{\sigma}=\left(\frac{\sigma_{M C S}-\sigma_{2 P E M}}{\sigma_{M C S}}\right) \times 100
\end{gathered}
$$

Table $\mathrm{V}$ indicates that the 2PEM can decrease the run time severely and still maintain a high level of accuracy. The time efficiency of the 2PEM is decreased as the number of uncertain variables increases, because the deterministic LF is ran twice for each uncertain variable, once for the value below the mean, and once for the value above the mean, with other variables kept at their means. Therefore, the 2PEM run time is proportional to the number of uncertain variables.

TABLE V. COMPARISON OF ACCURACY AND COMPUTATIONAL EFFORTS OF DIFFERENT METHODS

\begin{tabular}{|l|c|c|c|c|}
\hline \multirow{2}{*}{ Case study } & METHOD & RUN TIME[s] & $\varepsilon_{\mu}[\%]$ & $\varepsilon_{\sigma}[\%]$ \\
\hline \multirow{3}{*}{ Case 1} & MCS & 9.864 & 0 & 0 \\
\cline { 2 - 5 } & 2 PEMS & 0.18 & 1.9 & 9.68 \\
\cline { 2 - 5 } & 2 PEMU & 0.188 & 0.038 & 0.76 \\
\hline \multirow{3}{*}{ Case 2 } & MCS & 28.76 & 0 & 0 \\
\cline { 2 - 5 } & 2PEMS & 0.884 & 0.194 & 2.89 \\
\cline { 2 - 5 } & 2PEMU & 0.884 & 0.181 & 0.716 \\
\hline
\end{tabular}

\section{CONCLUSIONS}

The incorporation of renewable energy sources into power systems is constantly increasing, as a result of energy and environmental concerns. Renewable energy sources are variable and intermittent and introduce uncertainties into power systems. Probabilistic methods are employed in order to analyze these uncertainties. In this paper, the Two Point Estimation Method is proposed for Probabilistic Load Flow study. Comparison of the results obtained by utilizing two 
different methods indicates that the Two Point Estimation Method shows a similar level of accuracy and a smaller computational burden compared to the Monte Carlo Simulation method. Results also indicate that the unsymmetrical Two Point Estimation Method shows a higher level of accuracy compared to the symmetrical one, with equal computing effort. The time efficiency of the Two Point Estimation Method is decreased with the increase of the number of uncertain variables since the run time for this method is proportional to the number of uncertain variables.

\section{APPENDIX}

The detailed technical data about used WTGs and wind speed data is given in Table VI.

TABLE VI. WIND FARM INFORMATION

\begin{tabular}{|c|c|}
\hline Parameters & VALUE \\
\hline No. of WTGs & 25 \\
\hline$P_{r}[\mathrm{MW}]$ & 0.6 \\
\hline $\mathrm{V}_{\mathrm{r}}[\mathrm{m} / \mathrm{s}]$ & 15 \\
\hline $\mathrm{V}_{\mathrm{i}}[\mathrm{m} / \mathrm{s}]$ & 3 \\
\hline $\mathrm{V}_{\mathrm{o}}[\mathrm{m} / \mathrm{s}]$ & 25 \\
\hline $\mathrm{C}[\mathrm{m} / \mathrm{s}]$ & 6.7703 \\
\hline $\mathrm{K}$ & 2 \\
\hline
\end{tabular}

\section{REFERENCES}

[1] B. Borkowska, "Probabilistic Load Flow", IEEE Trans. Power App. and Syst., Vol. PAS-93, No. 3, pp. 752-759, 1974.
[2] P. Jorgensen, J. Christensen, J. Tande, "Probabilistic Load Flow Calculation Using Monte Carlo Techniques for Distribution Network with Wind Turbines", in Proc. 8th International Conf. Harmonics and Quality of Power, Vol. 2, pp. 1146 -1151, Athens, Greece, 1998.

[3] P. Zhang, S. Lee, "Probabilistic Load Flow Computation Using the Method of Combined Cumulants and Gram-charlier Expansion", IEEE Trans. Power Syst., Vol. 19, No. 1, pp. 676 - 682, 2004.

[4] D. Lei, Z. Chuan-cheng, Y. Yi-han, Z. Pei, "Improvement of Probabilistic Load Flow to Consider Network Configuration Uncertainties", APPEEC Power and Energy Engineering. Conf., AsiaPacific, pp. 1-5, Wuhan, 2009.

[5] L. Dong, W. Cheng, H. Bao, Y. Yang, "Probabilistic Load Flow Analysis for Power System Containing Wind Farms", APPEEC Power and Energy Engineering Conf., Asia-Pacific, pp. 1-4, Chengdu, 2010.

[6] R. Allan, A. Leite da Silva, R. Burchett, "Evaluation Methods and Accuracy in Probabilistic Load Flow Solutions", IEEE Trans. Power App. and Syst., Vol. PAS-100, No. 5, pp. 2539-2546, 1981.

[7] L. Hong, L. Shi, L. Yao, Y. Ni, M. Bazargan, "Study on Fuzzy Load Flow with Consideration of Wind Generation Uncertainties", Transmission Distribution Conference Exposition: Asia and Pacific, 2009, pp. $1-4,2009$.

[8] H. P. Hong, "Discussion on "An Optimal Point Estimate Method for Uncertainty Studies" by J. He and G. Sallfors", App. Math. Modeling, Vol. 19, No. 8, pp. 508-509, 1995.

[9] H. P. Hong, "An Efficient Point Estimate Method for Probabilistic Analysis", Reliability Engineering and System Safety, Vol. 59, No. 3, pp. $261-267,1998$.

[10] C. - L. Su, "Probabilistic Load Flow Computation Using Point Estimate Method", IEEE Trans. Power Syst., Vol. 20, No. 4, pp. 1843-1851, 2005.

[11] M. Basil, A. Jamieson, "Uncertainty of Complex Systems by Monte Carlo Simulation", 16th North Sea flow measurement workshop, Gleneagles, pp. 1-10, 1998.

[12] G. Verbic, C. A. Canizares, "Probabilistic Optimal Power Flow in Electricity Markets Based on a Two-Point Estimate Method", IEEE Trans. Power Syst., Vol. 21, No. 4, pp. 1883-1893, 2006. 